\section{Serologische Tumormarker beim malignen Melanom}

U. Wollina, Kerstin Karte, Uta-Christina Hipler

Klinik für Dermatologie und Allergologie, Klinikum der Friedrich-Schiller-Universität Jena (Direktor: Prof. Dr. med. P. Elsner)
Zusammenfassung. Das maligne Melanom der Haut ist aufgrund der epidemiologischen Situation und der erheblichen stadienabhängigen Prognose von besonderer Relevanz für die Dermato-Onkologie. Die vorliegende Übersicht widmet sich den Möglichkeiten der serologischen Tumormarker-Analyse und ihrer praktischen Bedeutung in der Melanomdiagnostik und -nachsorge. Im einzelnen werden die Superoxiddismutasen (SOD), das Interzelluläre Adhäsionsmolekül-1 (ICAM-1) und S$100 \beta$ besprochen. Bezüglich der SOD sind sowohl MnSOD als auch CuZnSOD untersucht worden. Nur für die CuZnSOD liegen Befunde im humanen System vor, die keinen Bezug zum Tumorstadium oder der Prognose erkennen lassen. ICAM-1 ist ein in allen Tumorstadien ansprechender Melanommarker, der auch eine Prognoserelevanz aufweist. Nachteilig ist die fehlende Melanomspezifität. S-100 $\beta$ zeigt keine Beziehung zu den frühen Tumorstadien, steigt jedoch deutlich in den Stadien III-IV (DDG) an, wenn noch eine Tumorlast nachweisbar ist. Es besteht eine hohe Spezifität für den Tumornachweis beim fortgeschrittenen malignen Melanom. Die diagnostische Effizienz ist jedoch geringer als bei modernen bildgebenden Verfahren wie der Positronen-Emissions-Tomographie (PET).

Serological Tumor Markers in Malignant Melanoma. Both the epidemiological situation and the stage-dependent prognosis contribute to the importance of malignant melanoma for dermato-Oncology. The present review contributes to serological tumor marker analysis and the practical input in diagnostics and follow-up. The paper discusses superoxide dismutases (SOD), intercellular adhesion molecule-1 (ICAM-1), and S-100 $\beta$. Among the SOD's MnSOD and CuZnSOD are of interest, but data are available only for CuZnSOD in the human system. The enzyme does not show any correlation to either tumor stage or prognosis. ICAM-1 is detectable in all tumor stages with a relationship to prognosis. A major disadvantage of ICAM-1 is its non-specificity for melanoma. $S-100 \beta$ does not correspond to lower tumor stages, but the level markedly increases in stages III and IV (DDG), when a tumorload is evident. It shows a high specificity for tumor presence in advanced melanoma. Its diagnostic efficacy, however, is low compared to imaging techniques such as positron emission tomography (PET).

\section{Einführung}

Die frühe Diagnosestellung ist für die Prognose beim malignen Melanom (MM) von herausragender Bedeutung. Wichtigster Prognose-Parameter ist die Tumordicke nach Breslow. Patienten mit einer lokoregionalen oder Fernmetastasierung weisen eine Prognoseverschlechterung auf [9]. Neben bildgebenden Verfahren, deren Empfindlichkeit und Aussagekraft in den letzten Jahren deutlich gewonnen hat [12], wurde seit langem versucht, serologische Tumormarker zu nutzen.

Laborbestimmungen von Serum-Lactatdehydrogenase (LDH), 5-S-Cysteinyl-DOPA und neuronenspezifischer Enolase sind in der Vergangenheit mit wechselndem Erfolg verwendet worden. Nach anfänglicher Euphorie wurden die Verfahren in praxi wieder verlassen bzw. konnten sich erst gar nicht allgemein durchsetzen.

In jüngster Zeit wurden neue Marker herangezogen, von denen im Folgenden die Superoxiddismutasen (SOD), das Interzelluläre Adhäsionsmolekül-1 (ICAM-1) und S-100 $\beta$ besprochen werden sollen.

\section{Superoxiddismutasen}

Die SOD's sind Metalloproteine, die die Umwandlung/Dismutation von $\mathrm{O}_{2}-\mathrm{zu} \mathrm{O}$ und $\mathrm{H}_{2} \mathrm{O}_{2}$ katalysieren. Sie schützen die sauerstoff-metabolisierenden Zellen vor direkten und indirekten Schädigungen durch Radikale. Die Mn-abhängige SOD ist in der mitochondrialen Matrix lokalisiert. Bei murinen B16Melanomen sind die aggressiveren Varianten mit hoher Metastasierungspotenz durch einen Mn-SOD-Mangel gekennzeichnet [10]. Für den Menschen liegen bisher keine publizierten Daten vor.

Die CuZnSOD findet sich vorwiegend im Zytoplasma. Es kann durch Zytokine wie TNF $\alpha$ oder Interleukin-1 induziert werden. Wir untersuchten dieses Enzym mittels ELISA (Bender MedSystems, Wien) bei über $200 \mathrm{MM}$-Patienten und fanden weder eine Verminderung bei MM-Patienten noch eine stadienabhängige Beziehung der CuZnSOD-Konzentration [17].

Akt Dermatol 2001; 27: 230-232 


\section{Lösliches ICAM-1}

ICAM-1 ist ein immunglobulin-artiges Molekül, das auch von Melanomzellen exprimiert werden kann. Es wird durch Zytokine wie TNF $\alpha$, Interleukin-1 und Interferon $\gamma$ induziert. ICAM1 spielt eine Rolle bei T-zellulär vermittelten Abwehrmechanismen, im Rahmen der Entzündung und der Tumorimmunität [2]. Behandlung von Melanomen mit Antisense-Oligonukleotiden von ICAM-1 erhöht die Metastasierungspotenz TNF $\alpha$-vorbehandelter Melanome [11]. Die Transfektion von Melanomzellen mit dem Tumorsuppressor-Gen nm23 vermindert das Ablösen von ICAM-1 (shedding) von Melanomzellen und verringert so den Spiegel an löslichem (s)ICAM-1. Im Tiermodell werden auf diese Weise zytotoxische Tumorabwehrmechanismen gefördert [13]. In eigenen Untersuchungen an 265 MMPatienten und 930 Serumproben mittels sensitivem ELISA (R \& D Deutschland, Wiesbaden) wurden signifikant höhere sICAM-1 Konzentrationen in allen Tumorstadien im Vergleich zur gesunden Kontrollgruppe gemessen $(p<0,0005)$ [17]. Außerdem wurde eine stadienabhängige Zunahme der Werte festgestellt (vgl. $[8,14])$. Unabhängig vom Tumorstadium zeigten MM-Patienten mit normalem sICAM-1 eine signifikant bessere Überlebensrate über 32 Monate (100\%) als solche mit erhöhten Spiegeln (88\%) [17].

Nachteilig ist die geringe Spezifität dieses Markers für das Melanom. Erhöhte Werte sind u.a. bei Lymphomen und zahlreichen entzündlichen Erkrankungen (Rheumatoidarthritis, Nephropathien, multiple Sklerose u.a.m.) zu finden.

\section{Serum S-100ß}

Das S-100-Protein ist ein dimeres kalziumbindendes Eiweiß von $21 \mathrm{Kda}$, das aus $\alpha$ - und $\beta$-Untereinheiten besteht. Es existieren 3 Isoformen: S-100 $\alpha \beta$, S-100 $\alpha \alpha$ und S-100 $\beta$. Das Protein kommt in hohen Konzentrationen im ZNS und in Pigmentzellen einschließlich der MM vor. Die Immunohistochemie mit polyklonalen Antikörpern gegen S-100 ist mittlerweile Routine für die Melanomdiagnostik [5,16].

Wir untersuchten Serum-S-100 $\beta$ bei 315 MM-Patienten mit einem sensitiven immunluminometrischen Assay (LIA-mat Sangtec100, Byk-Sangtec Diagnostica, Dietzenbach). Die tumorfreien Patienten $(n=310)$ der Stadien Ia bis IV (DDG) zeigten einen S-100ß-Mittelwert von $0,064 \mu \mathrm{g} / \mathrm{L}$, was sich nicht signifikant von den Kontrollen unterschied. Bei Patienten mit fortgeschrittenem MM und Tumorlast ( $n=61$; Tumorstadien IIIa bis IV) war der Wert auf 0,605 $\mu \mathrm{g} / \mathrm{L}$ im Mittel erhöht. Für die Stadien IIIb und IV erreichten die Mittelwertunterschiede zu den Kontrollen Signifikanz ( $p<0,0001$; Abb.1). Erhöhte S$100 \beta$-Werte $>0,120 \mu \mathrm{g} / \mathrm{L}$ erreichten eine Sensitivität von $70,8 \%$ bei einer Spezifität von $89,9 \%$ für den Tumornachweis (Tumorlast) [19]. Eine Beziehung von Tumorstadium bzw. Prognose und erhöhtem S-100 $\beta$ im Serum wurde auch von anderen Autoren nachgewiesen [1,3,4,6,7,12,15].

Hingegen kamen Buer et al. (1997) bei einer multivariaten Cox Proportional-Hazard-Analyse bei 99 Patienten mit metastasierendem MM zur Schlussfolgerung, dass S-100 $\beta$ keine Zusatzinformation zu bekannten Prognoseparametern lieferte [4]. Einschränkend ist jedoch anzumerken, dass die Autoren einen extrem hohen Cutt-off-Level von 3,0 $\mu \mathrm{g} / \mathrm{L}$ verwendeten, der das 15 fache des Üblichen beträgt.

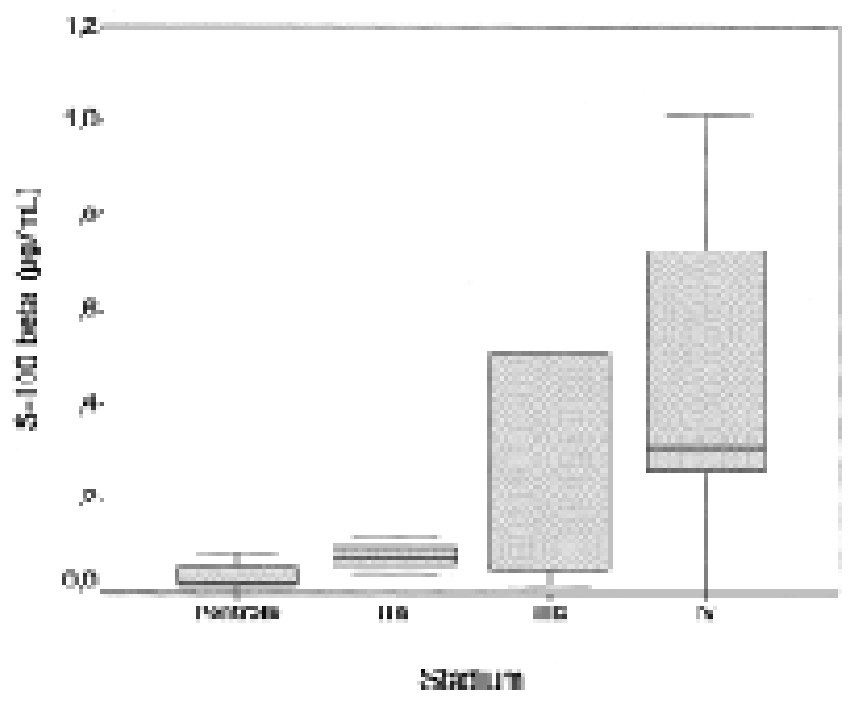

Abb. 1 S-100ß im Serum bei Patienten mit fortgeschrittenem Melanom und Tumorlast vs. Kontrollen.

Erhöhte S-100ß-Werte stellten wir bei 36 von 62 Proben von MM-Patienten im Stadium IV fest (58,1\%). Der Prozentsatz war bei Patienten mit Tumorlast oder in Progression mit $94,4 \%$ erheblich größer als bei solchen in klinischer Remission $(7,7 \%)$.

\section{Ausblick}

Die Situation der Tumormarker-Bestimmung ist für das MM noch immer unbefriedigend. Trotz punktueller Fortschritte fehlen bislang noch preisgünstige, schnelle und verlässliche Testverfahren für melanom-spezifische Moleküle, die die Sensitivität in Diagnostik und Nachsorge signifikant erhöhen. Wir erachten die Bestimmung von S-100 $\beta$ im Serum bei Patienten mit fortgeschrittenen Melanomen zur Rezidiverkennung als überprüfenswert. Im Rahmen von kontrollierten Therapiestudien ist die Verwendung zum Monitoring aussichtsreich. Es bedarf jedoch weiterer Anstrengungen, den „idealen“ Tumormarker für das MM zu definieren.

\section{Literatur}

${ }^{1}$ Abraha HD, Fuller LC, Du Vivier AWP, Higgins EM, Sherwood RA. Serum S-100 protein: a potentially useful prognostic marker in cutaneous melanoma. Br J Dermatol 1997; 137: 381 - 385

2 Becker JC, Termeer C, Schmidt RE, Bröcker EB. Soluble intercellular adhesion molecule-1 inhibitis MHC-restricted specific T cell/ tumor interactions. J Immunol 1993; 151: 7224-7232

${ }^{3}$ Bronfrèr JMG, Korse CM, Nieweg OE, Rankin EM. The luminescence immunoassay S-100: a sensitive test to measure circulating S-100 $\beta$ : its prognostic value in malignant melanoma. $\mathrm{Br} \mathrm{J}$ Cancer 1998; 77: 2210-2214

${ }^{4}$ Buer J, Probst M, Franzke A, Duesing S, Haindl J, Volkenandt M, Wittke F, Hoffmann R, Ganser A. Eleveated serum levels of S100 and survival in metastatic malignant melanoma. Br J Cancer 1997; 75: 1373 - 1376

${ }^{5}$ Cochran AJ, Lu HF, Li PX, Saxton R, Wen DR. S-100 protein remains a practical marker for melanocytic lesions and other tumors. Melanoma Res 1993; 3: 325 - 330 
${ }^{6}$ Hauschild A, Engel G, Brenner W, Gläser R, Mönig H, Henze E, Christophers E. S100 $\beta$ protein detection in serum is a significant prognostic factor in metastatic melanoma. Oncology 1999; 56: $338-344$

${ }^{7}$ Henze G, Dummer R, Joller-Jemelka HI, Böni R, Burg G. Serum S100 - a marker for disease monitoring in metastatic melanoma. Dermatology 1997; 194: 208 - 212

${ }^{8}$ Hirai S, Kageshita T, Kimura T, Tsujikasi M, Imai K, Wakamatsu K, Ito S, Ono T. Serum levels of sICAM-1 and 5-cysteinyldopa as markers of melanoma progression. Melanoma Res 1997; 7: 58 - 62

${ }^{9}$ Kaufmann R, Tilgen W, Garbe C. Malignes Melanom. Hautarzt 1998; 48 (Suppl 1): 30-38

${ }^{10}$ Kwee JK, Mitidieri E, Affonso OR. Lowered superoxide dismutase in highly metastatic B16 melanoma cells. Cancer Lett 1991; 57: 199-202

${ }^{11}$ Miele ME, Bennett CF, Miller BE, Welch DR. Enhanced metastatic ability of TNF-alpha-treated malignant melanoma cells is reduced by intercellular adhesion molecule-1 (ICAM-1, CD54) antisense oligonucleotides. Exp Cell Res 1994; 214: 231 - 241

12 Mruck S, Baum RP, Rinne D, Hor G. Diagnostic accuracy and predictive value of the tumor-associated antigen S100 in malignant melanomas: validation by whole body FDG-PET and conventional diagnostics. Anticancer Res 1999; 19: 2685-2690

13 Parhar RS, Shi Y, Zou M, Farid NR, Ernst P, Al-Sedairy ST. Effects of cytokine-mediated modulation of $\mathrm{nm} 23$ expression on the invasion and metastatic behaviour of B16F10 melanoma cells. Int J Cancer 1995; 60: 204-210

${ }^{14}$ Schaider H, Rech-Weichselbaum I, Richtig E, Seidl H, Soyer HP, Smolle J, Kerl H. Circulating adhesion molecules as prognostic factors for cutaneous melanoma. J Am Acad Dermatol 1997; 36: $209-213$

15 Schultz ES, Diepgen TL, von den Driesch P. Clinical and prognostic relevance of serum S-100beta protein in malignant melanoma. $\mathrm{Br}$ J Derm 1998; 138: $426-430$

${ }^{16}$ Wollina U, Kilian U, Henkel U, Schaarschmidt H, Knopf B. The initial steps of tumor progression in the melanocytic lineage - a histochemical approach. Anticancer Res 1991; 11: 1405-1414

17 Wollina U, Karte K, Olbertz K, Hipler UC. Simultaneous measurement of circulating intercellular adhesion molecule- 1 and serum copper zinc dismutase activity in patients with malignant melanoma. Oncol Rep 1998; 5: 473-476

${ }^{18}$ Wollina U, Karte K, Hipler UC, Olbertz K. Soluble ICAM-1 seems to be related to prognosis in malignant melanoma. J Eur Acad Dermatol Venereol 1999; 12: 264-266

${ }^{19}$ Wollina U, Karte K, Hipler UC, Knöll B, Kirsch K, Herold C. Serum protein S-100beta in patients with malignant melanoma detected by an immunoluminometric assay. J Cancer Res Clin Oncol 2000; 126: $107-110$
Prof. Dr. U. Wollina

Hautklinik

Krankenhaus Dresden-Friedrichstadt

Friedrichstraße 41

01067 Dresden 